\title{
Evaluation of prognosis of squamous cell carcinoma of the oesophagus by endoscopic ultrasonography
}

\author{
M Shinkai, Y Niwa, T Arisawa, N Ohmiya, H Goto, T Hayakawa
}

\begin{abstract}
Backgroundlaims-For pretherapeutic staging of squamous cell carcinoma of the oesophagus, endoscopic ultrasonography (EUS) is considered the most profitable modality because it can provide cross sectional imaging of the tumour. The aim of this study was to evaluate the relation between prognosis and EUS findings, especially tumour area, in squamous cell carcinoma of the oesophagus.

Patients/methods-A total of 113 patients with squamous cell carcinoma of the oesophagus underwent EUS for pretherapeutic examination at Nagoya University Hospital. We compared EUS findings, histological results, and outcome. In addition, we measured the area of the tumour on EUS images $(n=113)$ and evaluated if EUS area correlated with volume of the tumour on histological findings $(n=50)$.

Results-The overall accuracy rate of EUS was $83.2 \%$ (94/113) for depth of tumour invasion and $67.6 \%(69 / 102)$ for perioesophageal lymph node metastasis. The EUS area increased in proportion to the development of tumour infiltration, and patients with lymph node metastasis had a larger EUS area than patients without lymph node metastasis. There was a close correlation between EUS area and volume of the tumour on histological findings. If EUS area of the tumour was less than $50 \mathrm{~mm}^{2}$, the five year survival rate was $100 \%$. As EUS area increased, the survival rate decreased.

Conclusions-Measurement of EUS area of the tumour is reliable for quantification of the tumour and prediction of prognosis in patients with squamous cell carcinoma of the oesophagus.

(Gut 2000;47:120-125)
\end{abstract}

Keywords: squamous cell carcinoma; oesophagus; endoscopic ultrasonography; prognosis; preoperative staging

The prognosis of oesophageal cancer patients is poor because the majority of lesions are in the advanced stage at the time of diagnosis. ${ }^{1}$ Recently however, because of more widespread knowledge of early stage oesophageal cancer and the use of videoendoscopy with Lugol staining for high risk patients, it has been possible to detect oesophageal cancer at earlier stages. ${ }^{2-4}$ If we can detect oesophageal cancer in the mucosal stage, we can use endoscopic mucosal resection, which is effective and the minimum local treatment. ${ }^{56}$ Recently, endoscopic mucosal resection (EMR) has become widely used in Japan, and contributes to the improved quality of the patient's life after treatment.

Endoscopic ultrasonography (EUS) can provide cross sectional imaging of the gastrointestinal wall and adjacent structures ${ }^{7-9}$ and thus provide information on choice of treatment and evaluation of prognosis in oesophageal cancer. We believe that EUS is an essential part of the pretherapeutic examination for staging of oesophageal cancer. To make the most of the specific characteristics of EUS, we measured the area of the tumour on EUS images and assumed that the tumour area on the EUS image was generally in proportion to the volume of the tumour. As the relation between EUS findings and prognosis of oesophageal cancer patients is not well known, the aim of this study was to evaluate the relation between prognosis and EUS findings, especially tumour area, in squamous cell carcinoma of the oesophagus.

\section{Materials and methods}

PATIENTS

From January 1988 to December 1997, 319 patients with oesophageal cancer underwent EUS for pretherapeutic examination at the Second Department of Internal Medicine, Nagoya University Hospital. In 113 of 319 patients it was possible to compare EUS findings and histopathological results, with obvious outcome after treatment. The remaining 206 patients were excluded from the study because of chemotherapy or radiation therapy before operation, follow up in another institution, inoperable lesions, or death from another disease. A total of 113 patients (99 men, 14 women; mean age 60 years, range 42-78) underwent EMR $(n=11)$ or surgery $(n=102)$ for initial treatment at our institution. The observation period after treatment was 30.1 (2.2) months (mean (SEM)). All were found to have histopathologically confirmed squamous cell carcinoma.

For pretherapeutic evaluation, we examined invasion of the tumour and perioesophageal lymph node metastasis with EUS. We performed EUS using the water infusion or water filled balloon method. In all cases, EUS findings were compared with histopathological findings of resected specimens. Histopathological evaluation was based on guidelines estab-

Abbreviations used in this paper: EUS, endoscopic ultrasonography; EMR, endoscopic mucosal resection. 


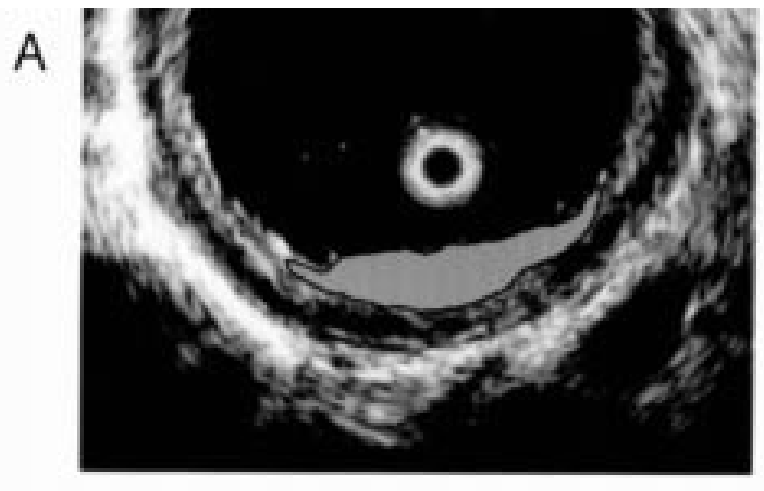

B

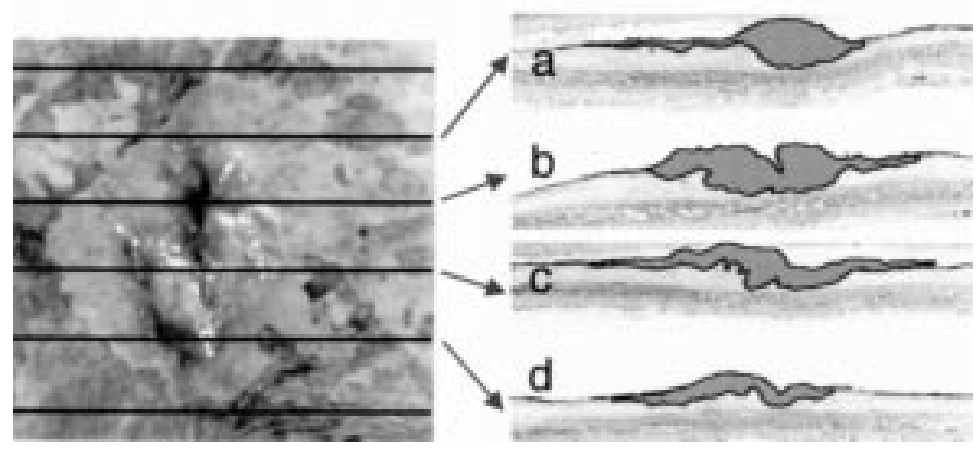

Figure 1 Measurement of tumour area, by endoscopic ultrasonography (EUS), and pathological tumour volume. (A) Ultrasound probe image of submucosal cancer. The ultrasound probe reveals a hypoechoic mass region extensively invading into the high echoic third layer (submucosal layer). In this case, the EUS area of the tumour was $36.1 \mathrm{~mm}^{2}$. (B) Histological findings after surgery showed the tumour limited to the submucosa (haematoxylin-eosin, original magnification). Pathological tumour volume $\left(\mathrm{cm}^{3}\right)$ is the total tumour area $(a+b+c+d)\left(\mathrm{cm}^{2}\right) \times$ width of prepared specimen $(0.4 \mathrm{~cm})$ In this case, the pathological tumour volume was $3.20 \mathrm{~cm}^{3}$.

lished by the Japanese Society for Esophageal Disease. ${ }^{10}$ There were no complications in this study.

INSTRUMENTS

We used the radial sector scanners GF-UM2 (7.5 MHz), GF-UM20 (switchable 7.5 MHz and $12 \mathrm{MHz}$ ), and GF-UM200 (switchable 7.5 $\mathrm{MHz}$ and $20 \mathrm{MHz}$; Olympus Optical Co. Ltd, Tokyo, Japan), and the ultrasound probes

Table 1 Patient characteristics

\begin{tabular}{lrrrrr}
\hline & \multicolumn{3}{c}{ Depth of tumour invasion } & \\
\cline { 2 - 5 } & $p M$ & $p S M$ & $p M P$ & $p$ Ad+Adjd & Total \\
\hline Number of patients & 19 & 34 & 18 & 42 & 113 \\
Location of the lesion & 0 & 5 & 2 & 7 & 14 \\
$\quad$ Proximal (Ce-Ut) & 12 & 19 & 9 & 20 & 60 \\
$\quad$ Middle (Mt) & 7 & 10 & 7 & 15 & 39 \\
$\quad$ Distal (Lt-Ae) & 19 & 34 & 0 & 0 & 53 \\
Macroscopic findings & 0 & 0 & 4 & 2 & 6 \\
$\quad$ Superficial type & 0 & 0 & 12 & 27 & 39 \\
$\quad$ Protruding type & 0 & 0 & 2 & 11 & 2 \\
$\quad$ Ulcerative and localised type & 0 & 0 & 0 & 2 & 54 \\
$\quad$ Ulcerative and infiltrating type & 0 & 16 & 8 & 30 & 48 \\
$\quad$ Diffusely infiltrating type & 8 & 18 & 10 & 12 & 11 \\
Lymph node metastasis & & & & \\
$\quad$ Positive & 11 & 0 & 0 & 0 & 102 \\
$\quad$ Negative & 8 & 34 & 18 & 42 & \\
Therapy & & & & \\
$\quad$ EMR & & & & & \\
$\quad$ Operation & & & & & \\
\hline
\end{tabular}

p, pathological findings; $M$, tumour invades mucosa; SM, tumour invades submucosa; MP, tumour invades musclaris propria; $\mathrm{Ad}+\mathrm{Adj}$, tumour invades adventitia or adjacent structures.

$\mathrm{Ce}$, cervical oesophagus; Ut, upper thoracic esophagus; Mt, middle thoracic oesophagus; Lt, lower thoracic esophagus; Ae, abdominal oesophagus.

EMR, endoscopic mucosal resection.
MP-PN15-08M (15 MHz; Aloka Co. Ltd, Tokyo, Japan), UM-2R (12 MHz) and UM-3R (20 MHz; Olympus Optical Co. Ltd).

PRETHERAPEUTIC STAGING (DEPTH OF TUMOUR INVASION AND PERIOESOPHAGEAL LYMPH NODE METASTASIS) BY EUS

We performed pretherapeutic staging of oesophageal cancer by EUS. For evaluation of the depth of tumour invasion by EUS, we used diagnostic criteria based on hypoechoic tumour area and oesophageal wall structure which was destroyed by tumour infiltration. Interpretation of EUS findings of normal oesophageal wall was guided by previous reports. ${ }^{89}$ For assessment of perioesophageal lymph node metastasis, we investigated all 102 patients who underwent open surgery with perioesophageal lymph node dissection. For determination of perioesophageal lymph node metastasis by EUS, a sharply rounded node with a size greater than $1 \mathrm{~cm}$ in diameter and a hypoechoic internal echo was considered a positive metastatic node. ${ }^{11}$

\section{MEASUREMENT OF AREA AND VOLUME OF THE} TUMOUR

After pretherapeautic staging, the maximum area of the tumour was measured on the EUS image in which the tumour as a low echoic area appeared largest. Firstly, for fundamental examination, we evaluated if the EUS area correlated with the volume of the tumour on histological findings. We measured the area of the tumour on sequentially prepared specimens of the entire tumour which was divided into rectangular slices $(45 \times 4 \mathrm{~mm})$, and calculated volume of the tumour by integration of tumour area and width $(4 \mathrm{~mm})$ of the prepared specimen. It was possible to compare the EUS area and the resected specimen volume in 50 cases. All 50 prepared specimens were fixed by the same method, and we considered that shrinkage of tissue occurred to the same degree in all specimens. If we could not detect the tumour as a low echoic area on the EUS image, we arbitrarily took the EUS area of the tumour as $0 \mathrm{~mm}^{2}$. Then we evaluated EUS area in relation to depth of invasion and lymph node metastasis. A practical method of measurement of EUS area and pathological tumour volume is represented in fig 1. In this study, we digitised EUS images by digital scanner and captured them with Photoshop (Adobe Systems Incorporated). We then used NIH Image (Internet, NIFTY-Serve software, ftp://zippy. nimh. nih. gov/pub/nih-image/) for measurement of the area of tumours by EUS.

SURVIVAL OF PATIENTS

We evaluated the relation between EUS findings and prognosis of oesophageal cancer patients. Firstly, we calculated survival rates according to the EUS area of the tumour. Then we calculated survival rates according to histological and EUS findings on depth of tumour invasion and perioesophageal lymph node metastasis. 
Table 2 Comparison between pretherapeutic EUS findings and pathological diagnosis on depth of tumor invasion

\begin{tabular}{lcrrll}
\hline & \multicolumn{2}{l}{ EUS findings } & & \\
\cline { 2 - 5 } Pathological diagnosis & $e M$ & $e S M$ & $e M P$ & $e A d+$ Adj & Accuracy rate \\
\hline pM & 17 & 2 & & & $89.5 \%$ \\
pSM & 3 & 26 & 3 & 2 & $76.5 \%$ \\
pMP & & 2 & 10 & 6 & $55.6 \%$ \\
pAd+Adj & & & 1 & 41 & $97.6 \%$ \\
PPV (\%) & 85.0 & 86.7 & 71.4 & 83.7 & Overall accuracy rate \\
NPV (\%) & 97.8 & 90.4 & 91.9 & 98.4 & $83.2 \%(94 / 113)$
\end{tabular}

p, pathological findings; e, EUS findings; $M$, tumour invades mucosa; SM, tumour invades submucosa; MP, tumour invades musclaris propria; $\mathrm{Ad}+\mathrm{Adj}$, tumour invades adventitia or adjacent structures.

PPV, positive predictive value; NPV, negative predictive value.

Table 3 Comparison between pretherapeutic EUS findings and pathological diagnosis on perioesophageal lymph node metastasis

\begin{tabular}{lll}
\hline & \multicolumn{2}{l}{ EUS finding } \\
\cline { 2 - 3 } Pathological diagnosis & $e N(+)$ & $e N(-)$ \\
\hline $\mathrm{pN}(+)$ & 41 & 13 \\
$\mathrm{pN}(-)$ & 20 & 28 \\
Sensitivity & $75.9 \%(41 / 54)$ \\
Specificity & $58.3 \%(28 / 48)$ \\
Overall accuracy & $67.6 \%(69 / 102)$
\end{tabular}

p, pathological findings; e, EUS findings; $N$, lymph node metastasis.

STATISTICAL METHODS

Values are expressed as mean (SEM). The $\chi^{2}$ test was performed to investigate the difference in EUS areas according to depth of tumour invasion, and the Mann-Whitney $U$ test was performed to investigate the difference in EUS areas between positive and negative lymph nodes. Pearson's correlation coefficient was used for evaluation of the correlation between EUS area and pathological tumour volume. Survival curves were calculated using the Kaplan-Meier method. The log rank test was performed to investigate differences between survival curves. A p value less than 0.05 was considered significant.

\section{Results}

PATIENT CHARACTERISTICS

The characteristics of patients are listed in table 1. We followed Japanese guidelines on location of the tumour and macroscopic findings. ${ }^{10}$ Mucosal cancer patients had no lymph node metastasis and 11 of 19 patients were treated

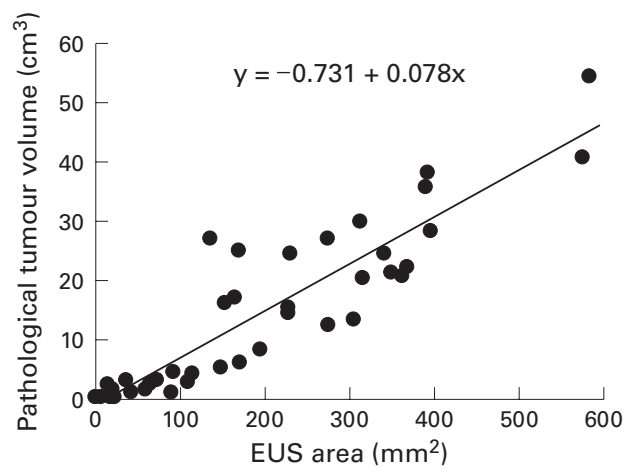

Figure 2 Correlation between area, measured by endoscopic ultrasonography (EUS), and pathological volume of the tumour. There was a high correlation between EUS area and pathological tumour volume. Pearson's correlation coefficient was 0.92 .
Table 4 EUS area of the tumour according to depth of tumour invasion and perioesophageal lymph node metastasis

\begin{tabular}{|c|c|}
\hline & $\begin{array}{l}\text { EUS area of the } \\
\text { tumour }\left(\mathrm{mm}^{2}\right)\end{array}$ \\
\hline \multicolumn{2}{|c|}{ Depth of tumour invasion } \\
\hline $\mathrm{pM}(\mathrm{n}=19)$ & $10.6(2.9)^{\star} \dagger$ \\
\hline $\mathrm{pSM}(\mathrm{n}=34)$ & $72.7(10.9)^{\star}+$ \\
\hline $\mathrm{pMP}(\mathrm{n}=18)$ & $250.2(21.9)^{\star} \neq \S$ \\
\hline $\mathrm{pAd}+\operatorname{Adj}(\mathrm{n}=42)$ & $336.6(23.2) \dagger \pm \S$ \\
\hline \multicolumn{2}{|c|}{ Perioesophageal lymph node metastasis } \\
\hline $\mathrm{pN}(+)(\mathrm{n}=54)$ & $271.9(23.9)$ \\
\hline $\mathrm{pN}(-)(\mathrm{n}=48)$ & $136.1(18.3)^{\star \star \star}$ \\
\hline
\end{tabular}

$\mathrm{p}$, pathological findings; $\mathrm{M}$, tumour invades mucosa; SM, tumour invades submucosa; MP, tumour invades the musclaris propria; Ad+Adj, tumour invades adventitia or adjacent structures; $\mathrm{N}$, lymph node metastasis

${ }^{\star} \mathrm{p}<0.05$ compared with $\mathrm{pAd}+\mathrm{Adj} ; \mathrm{tp}<0.05$ compared with $\mathrm{pMP} ; \neq \mathrm{p}<0.05$ compared with $\mathrm{pSM} ; \mathrm{\$ p}<0.05$ compared with $\mathrm{p} M$.

${ }_{\star \star \star} \mathrm{p}<0.001$ compared with $\mathrm{pN}(+)$.

Values are mean (SEM).

with EMR. For cases of tumour invading the submucosa, 16 of 34 patients $(47.1 \%)$ had lymph node metastasis and none underwent EMR. For cases of tumour invasion to the adventitia or adjacent structures, 30 of 42 patients $(71.4 \%)$ had lymph node metastasis.

EVALUATION OF DEPTH OF TUMOUR INVASION BY EUS

For evaluation of depth of tumour invasion, we compared EUS findings with histological findings. The results of EUS assessment of the depth of tumour invasion are summarised in table 2. The accuracy rate for depth of tumour invasion was $89.5 \%$ for mucosal cancer, $76.5 \%$ for submucosal cancer, $55.6 \%$ for tumours invaded to the muscularis propria, and $97.6 \%$ for tumours invaded to the adventitia or adjacent structures. The overall accuracy rate was $83.2 \%$ (94/113). Positive and negative predictive values are given in table 2 .

ASSESSMENT OF PERIOESOPHAGEAL LYMPH NODE METASTASIS BY EUS

For diagnosis of perioesophageal lymph node metastasis, we compared EUS findings with histological findings in all 102 patients who underwent open surgery with perioesophageal lymph node dissection. The results of EUS assessment of lymph node metastasis are summarised in table 3. The sensitivity of the EUS diagnosis for

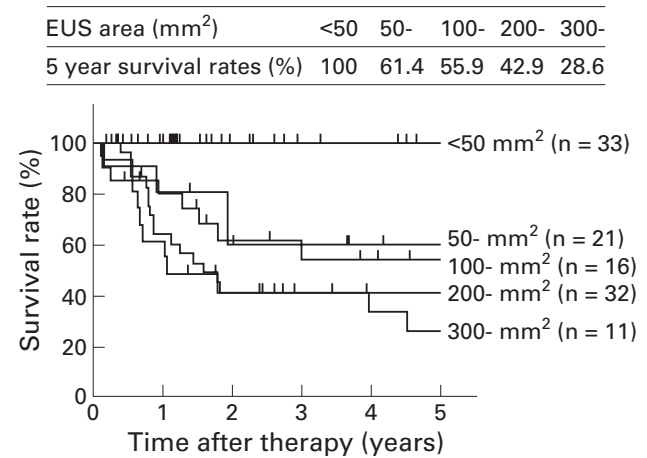

Figure 3 Kaplan-Meier survival curves for patients with oesophageal cancer, according to area, measured by endoscopic ultrasonography (EUS), of the tumour. Five year survival rates of all patients with a tumour area less than $50 \mathrm{~mm}^{2}$ on EUS findings was $100 \%$. 


\begin{tabular}{llllc}
\hline Pathology & pM & pSM & pMP & pAd + Adj \\
\hline $\begin{array}{l}5 \text { year } \\
\text { survival rates (\%) }\end{array}$ & 100 & 68.8 & 61.1 & 26.1 \\
\hline
\end{tabular}

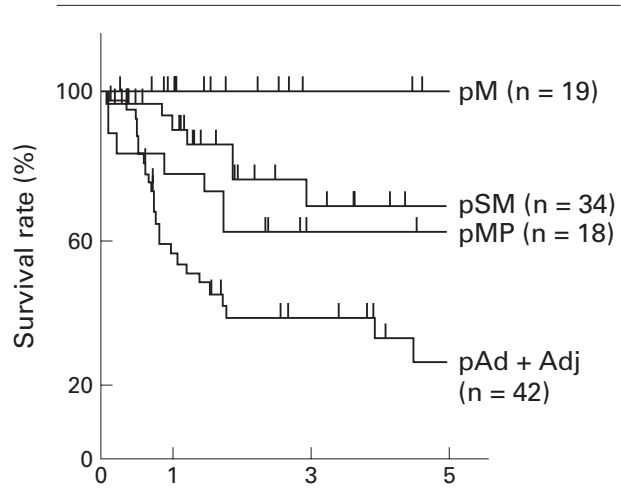

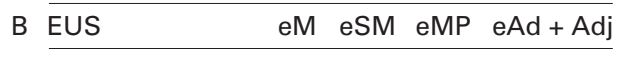

5 year 5 year
survival rates (\%) $100 \quad 73.0 \quad 55.6^{*} \quad 32.2$

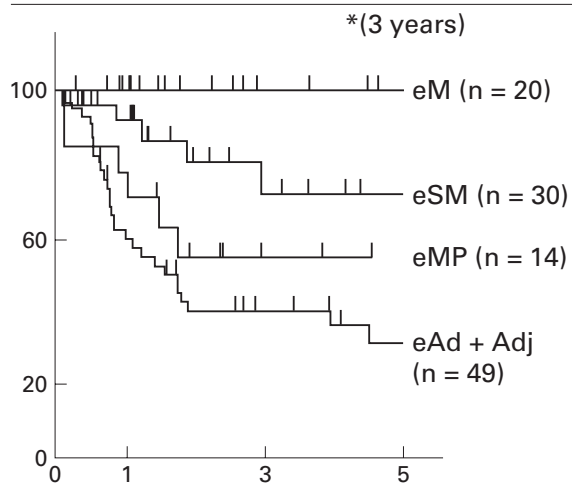

Time after therapy (years)

Figure 4 Kaplan-Meier survival curves for patients with oesophageal cancer according to depth of tumour invasion. (A) Survival curves according to histological diagnosis. (B) Survival curves according to endoscopic ultrasonography (EUS) findings.

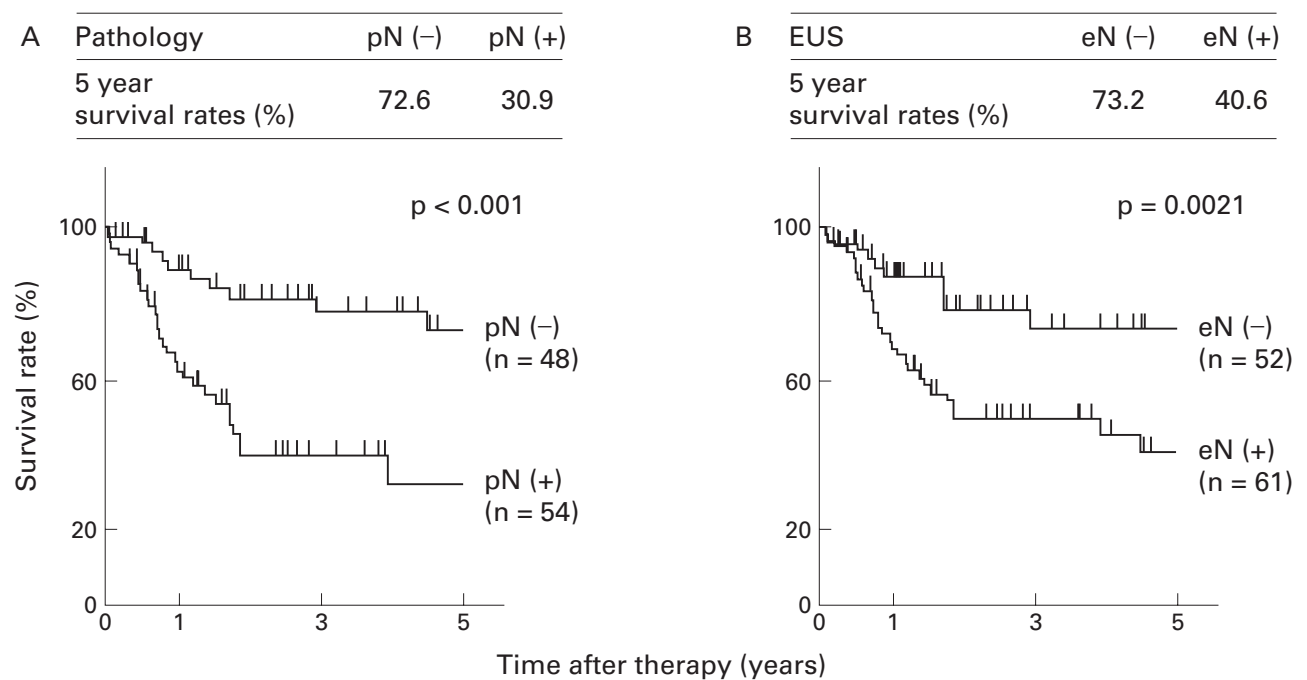

Figure 5 Kaplan-Meier survival curves for patients with oesophageal cancer according to perioesophageal lymph node metastasis. (A) Survival curves according to histological diagnosis. (B) Survival curves according to endoscopic ultrasonography (EUS) findings. There was a significant difference between positive and negative lymph node metastasis $((A) p<0.001,(B) p=0.0021)$.

lymph node metastasis was $75.9 \%(41 / 54)$ and specificity was $58.3 \%(28 / 48)$. The overall accuracy rate was $67.6 \%(69 / 102)$.

\section{AREA AND VOLUME OF THE TUMOUR}

We investigated the relation between EUS area and volume of the tumour calculated from the sequentially prepared specimens. The results of the correlation between EUS area and pathological volume of the tumour are displayed in fig 2. There was a close correlation between the two groups. The results of EUS area of the tumour according to depth of invasion and lymph node metastasis are shown in table 4 . EUS area increased in proportion to progression of tumour infiltration. Patients with lymph node metastasis had a significantly larger area than patients without lymph node metastasis $(\mathrm{p}<0.001)$.

SURVIVAL OF PATIENTS

The relation between patient survival and EUS area is displayed in fig 3 . When the EUS area of the tumour was smaller than $50 \mathrm{~mm}^{2}$, the five year survival rate was $100 \%$. As EUS area increased, the survival rate decreased. The prognosis of patients with an EUS area of more than $300 \mathrm{~mm}^{2}$ was very poor (five year survival rate $28.6 \%$ ). Figure 4 shows the survival of patients with oesophageal cancer related to depth of tumour invasion. In patients with mucosal cancer diagnosed by EUS or pathological findings, the five year survival rate was $100 \%$. With greater tumour infiltration, the survival rate decreased. Figure 5 shows survival of patients with oesophageal cancer related to perioesophageal lymph node metastasis. The survival rate of patients with lymph node metastasis was lower than that of patients without lymph node metastasis. There was a significant difference between the two groups.

\section{Discussion}

The prognosis of oesophageal cancer is poorer than that of other gastrointestinal cancers although many early stage oesophageal cancers 
have been detected recently owing to advances in diagnostic techniques. ${ }^{12} 13$ In our study, the five year survival rate of all patients was $54.5 \%$ although inoperable patients were excluded from the study. If advanced oesophageal cancer patients are operable, they undergo oesophagectomy with two or three field lymph node dissection. ${ }^{12}{ }^{13}$ Inoperable patients undergo various therapies such as chemotherapy, radiation therapy, laser therapy, or stenting for the purpose of palliation. At present, great efforts in the diagnosis and treatment of oesophageal cancer are being made towards improving the patient's quality of life.

For predicting the prognosis of oesophageal cancer patients, depth of tumour invasion and lymph node metastasis are the most important features. ${ }^{61415}$ Therefore, until now, we have made efforts to diagnose accurately these two areas. Based on our present data, when the tumour invaded the adventitia or adjacent structures, the five year survival rate was $26.1 \%$, and when lymph node metastasis was positive it was $30.9 \%$. In contrast, in patients with mucosal cancer, the five year survival rate was $100 \%$, and 11 of 19 cases $(57.9 \%)$ were treated with EMR. Therefore, accurate pretherapeutic staging is one of the most crucial points for prediction of prognosis and appropriate selection of treatment.

EUS is the most reliable method for local staging of oesophageal cancer. In evaluating the depth of tumour invasion, lymph node metastasis, and relation to adjacent structures, EUS is more accurate than computed tomography. ${ }^{816} 17$ In this study, the accuracy rates for EUS were $83.2 \%$ for diagnosis of depth of tumour invasion and $67.6 \%$ for evaluation of perioesophageal lymph node metastasis.

To date, we have performed staging of oesophageal cancer by tumour depth and lymph node metastasis. In this study, we concentrated on the ability of EUS to directly visualise the tumour on cross sectional images. Oesophageal cancer is not greatly affected by peptic ulcerative change as is gastric cancer and thus we were able to determine the oesophageal cancer tumour volume by EUS measurement of tumour area. Strictly speaking, the EUS area of the tumour is not equal to the area of the resected specimen, as in Japan EUS findings are shown as cross sectional images and histopathological findings give a longitudinal sectional picture ${ }^{10}$ For evaluation of gastric ulcer healing, we have previously proved that EUS images of resected ulcer specimens are equivalent to histological photographs. With respect to tumour area of the resected specimen, the expected contraction for specimens embedded in paraffin was $92 \%$ of the original area, and we could calculate a rough estimate of tumour volume by integration of tumour area and width of the prepared specimen. ${ }^{18}$ There have been few reports on EUS area of tumours until now, and even fewer reports that investigated the relation between EUS findings and prognosis of patients with oesophageal cancer. Isenberg et al reported that standard EUS staging is not accurate after neoadjuvant chemoradiation, and that measurement of maximal cross sectional area of the tumour is useful for assessing response of oesophageal cancer to preoperative chemoradiation. $^{19}$ In patients who have undergone preoperative chemoradiation, evaluation of depth of tumour invasion is difficult because of inflammation or fibrosis in the oesophageal wall. Thus in our study, patients treated with preoperative chemoradiation were excluded. For EUS assessment of response to chemoradiation, further studies are needed. In another report, Brugge et al found that EUS measurement of the maximal thickness of a malignant oesophageal mass can accurately predict extraoesophageal extension. ${ }^{20}$ In this way, EUS measurement of length and area of the tumour is very useful for assessment of diagnosis and treatment.

In the present study there was a close correlation between EUS area and volume based on histopathological findings. Measurement of EUS tumour area can be performed simply using existing EUS systems, and as EUS measurement of tumour area correlates closely with tumour volume on histopathological findings, it can substitute for tumour volume. From our results, prognosis of patients with an EUS area less than $50 \mathrm{~mm}^{2}$ was excellent but became worse with an increase in EUS area, indicating that tumour volume correlates with prognosis of patients with oesophageal cancer. We believe that this new method is simple and reliable for assessment of oesophageal cancer.

Erroneous diagnosis of depth of tumour invasion was caused by an undetectably small quantity of tumour invasion or inflammatory change in the submucosa. In our study, underdiagnosis of depth of tumour invasion was principally caused by a small quantity of tumour invasion into the submucosa. With the present level of EUS technology, we can diagnose cancer massively invading the submucosa but it is very difficult to detect minimal invasion of the submucosa. This change was extremely minute, and we do not believe it had a significant effect on measurement of EUS area of the tumour. Regarding evaluation of lymph node metastasis, specificity was low $(58.3 \%)$ because our diagnostic criteria are still insufficient. However, EUS area of the tumour in patients with positive lymph node metastasis was significantly larger than in patients with negative lymph node metastasis. Measurement of EUS area of the tumour might contribute to a diagnosis of lymph node metastasis.

Recently, three dimensional EUS was developed to visualise surface and internal structures of the tumour and its relation with adjacent structures; it is also applicable in the diagnosis of gastrointestinal tumours ${ }^{21}$ and can measure tumour volume. This system holds mind boggling possibilities but has not yet become widely used because of its high cost. We believe this technology can be used for diagnosis and judgment of treatment of oesophageal cancer in the future.

In conclusion, EUS and quantification of the EUS image are the most reliable modalities for preoperative staging and prediction of prognosis in oesophageal cancer because they provide an abundance of information on the depth of 
invasion, lymph node metastasis, tumour area, and volume.

1 Fockens P, Kisman K, Merkus MP, et al. The prognosis of esophageal carcinoma staged irresectable nosis of esophageal carcinoma staged irresectable 23.

2 Sugimachi K, Kitamura K, Baba K, et al. Endoscopic diagnosis of early carcinoma of the esophagus using Lugol's solution. Gastrointest Endosc 1992;38:657-61.

3 Meyer V, Burtin P, Bour B, et al. Endoscopic detection of early esophageal cancer in a high-risk population: does Lugol staining improve videoendoscopy? Gastrointest Endosc 1997;45:480-4.

4 Yokoyama A, Ohmori T, Makuuchi H, et al. Successful screening for early esophageal cancer in alcoholics using endoscopy and mucosa iodine staining. Cancer 1995;76: 928-34.

5 Makuuchi H. Endoscopic mucosal resection for early esophageal cancer-indication and techniques. Dig Endosc 1996;8:175-9.

6 Kodama M, Kakegawa T. Treatment of superficial cancer of the esophagus: A summary of responses to a questionnaire on superficial cancer of the esophagus in Japan. Surgery 1998;123:432-9.

7 Rösch T. Endoscopic ultrasonography. Endoscopy 1992;24: $144-53$.

8 Yoshikane H, Tsukamoto Y, Niwa Y, et al. Superficial esophageal carcinoma: evaluation by endoscopic ultrasonography. Am f Gastroenterol 1994;89:702-7.

9 Hasegawa N, Niwa Y, Arisawa T, et al. Preoperative staging of superficial esophageal carcinoma: comparison of an ultrasound probe and standard endoscopic ultrasonography. Gastrointest Endosc 1996;44:388-93.

10 Japanese Society for Esophageal Desease. Guidelines for clinical and pathologic studies on carcinoma of the esophagus, 9th edn. Tokyo: Kanehara publishing, 1992 (in Japanese).
11 Catalano MF, Sivak MV jr, Rice T, et al. Endosonographic features predictive of lymph node metastases. Gastrointest Endosc 1994;40:442-6.

12 Fujita H, Kakegawa T, Yamana H, et al. Mortality and morbidity rates, postoperative course, quality of life, and prognosis after extended radical lymphadenectomy for esophageal cancer. Ann Surg 1995;222:654-62.

13 Isono K, Sato H, Nakayama K. Results of a nationwide study on the three-field lymph node dissection of esophageal cancer. Oncology 1991;48:411-20.

14 Hiele M, Leyn PD, Schurmans P, et al. Relation between endoscopic ultrasound findings and outcome of patients with tumors of the esophagus or esophagogastric junction. Gastrointest Endosc 1997;45:381-6.

15 Chak A, Canto M, Gerdes H, et al. Prognosis of esophageal cancers preoperatively staged to be locally invasive (T4) by endoscopic ultrasound (EUS): a multicenter retrospective cohort study. Gastrointest Endosc 1995;42:501-6.

16 Tio TL, Cohen P, Coene PP, et al. Endosonography and computed tomography of esophageal carcinoma: preoperative classification compared to the new TNM system. Gastroenterology 1989;96:1478-86.

17 Botet JF, Lightdale CJ, Zauber AG, et al. Preoperative staging of esophageal cancer: comparison of endoscopic US and dynamic CT. Radiology 1991;181:419-25.

18 Niwa Y, Nakazawa S, Yoshino J, et al. Quantification of gastric ulcer healing by endoscopic ultrasonography. Gastrointest Endosc 1990;36:116-22

19 Isenberg G, Chak A, Canto MI, et al. Endoscopic ultrasound in restaging of esophageal cancer after neoadjuvant chemoradiation. Gastrointest Endosc 1998;48:158-63.

20 Brugge WR, Lee MJ, Carey RW, et al. Endoscopic ultrasound staging criteria for esophageal cancer. Gastrointest Endosc 1997;45:147-52.

21 Nishimura K, Niwa Y, Goto H, et al. Three-dimensional endoscopic ultrasonography of gastrointestinal lesions using an ultrasound probe. Scand $\mathcal{F}$ Gastroenterol 1997;32: $862-8$ 